\title{
Are all noisy quantum states obtained from pure ones?
}

\author{
Leah Henderson ${ }^{a}$, Noah Linden ${ }^{a}$ and Sandu Popescu ${ }^{b, c}$ \\ ${ }^{a}$ Department of Mathematics, University of Bristol, University Walk, Bristol, BS8 1TW, UK, \\ ${ }^{b} \mathrm{HH}$ Wills Physics Laboratory, University of Bristol, Tyndall Avenue, Bristol, BS8 1TL, UK, \\ ${ }^{c}$ BRIMS, Hewlett-Packard Laboratories, Stoke Gifford, Bristol BS12 6QZ, UK.
}

(11th April 2001)

\begin{abstract}
We ask what type of mixed quantum states can arise when a number of separated parties start by sharing a pure quantum state and then this pure state becomes contaminated by noise. We show that not all mixed states arise in this way. This is even the case if the separated parties actively try to degrade their initial pure state by arbitrary local actions and classical communication.
\end{abstract}

PACS numbers: 03.67-a, 03.65.Ud, 03.65.Ta

Density matrices and the question of their entanglement have been studied very intensively during the last few years [1]. However the question of how these density matrices arise in the first place has received much less attention. It has been tacitly assumed that density matrices arise when a number of parties, separated in space, start by sharing a pure state, and then this state gets contaminated by noise, due to interaction with the environment. Is it the case that any density matrix can be obtained in this way? Surprisingly we will show that the answer is no.

The point is that, in the above scenario, the very fact that we can talk about separated parties, means that the noise is local. This imposes constraints as to how entangled states may be degraded by the environment. We study here the effects of these constraints.

We will consider two different situations. The first is where we consider a pure state shared by the parties which is contaminated by local noise; this is a typical situation of obvious physical significance. We will refer to states which can be produced from pure states by local contamination as LC states. The second situation is one in which the parties actively try to degrade the pure state: in addition to local noise we allow local measurements and classical communication between the parties. We refer to states which can be produced from pure states by local contamination and classical communication as LCCC states.

We note that in both situations, when we wish to get a density matrix of a given number of parties each with a given dimension of local Hilbert space, we demand that the precursor pure states lie in a system Hilbert space of the same local dimensions [2].

The specific question we address is: given a general "target" density matrix, can we find a pure state from which this density matrix can be obtained by local con- tamination, in either situation. We will show that generically, in both situations, the answer is no.

In a sense, density matrices which can be obtained by local contamination are simple in that their entanglement properties are simply related to those of their pure state precursor. The density matrices which cannot be obtained in this way have a more subtle and complex structure.

Let us first discuss the situation in which no classical communication is allowed. We consider $n$ separated parties each of which has a $d$ level system. The number of real parameters describing pure states is $2 d^{n}-2$, and the number describing general mixed states is $d^{2 n}-1$. The fact that it is not possible to reach an arbitrary "target" density matrix by degrading any pure state follows simply from the fact that the number of parameters describing local degrading is linear in $n$. Thus for sufficiently large $n$, the number of parameters describing density matrices will be larger that the number describing pure states and local contamination.

In order to see how large $n$ needs to be we need to calculate the number of parameters describing the set of general local transformations. The most general interaction of a $d$-level system with its environment is as follows [3]:

$$
|i\rangle_{S}|0\rangle_{E} \mapsto\left|i^{\prime}\right\rangle_{S E}=\sum_{j}|j\rangle_{S}\left|e_{i j}\right\rangle_{E}
$$

where $|i\rangle_{S}$ are an orthonormal basis for the states of the system and $\left|e_{i j}\right\rangle_{E}$ are $d^{2}$ arbitrary (i.e. not necessarily normalised or orthogonal) states of the environment. It is only the norms and overlaps of the $\left|e_{i j}\right\rangle_{E}$ which are important and there are $d^{4}$ real parameters describing these. In fact the number of parameters describing the local degradation is less than $d^{4}$ since the $d^{2}$ environment states satisfy $d^{2}$ conditions arising from the fact the original states of the system $|i\rangle_{S}$ are an orthonormal basis.

Thus the total number of real parameters describing all pure states plus the number describing the local contamination is at most

$$
2 d^{n}-2+n\left(d^{4}-d^{2}\right) .
$$

This number is to be compared to the number of parameters describing density matrices of $n d$-level systems, namely $d^{2 n}-1$.

In fact the number of parameters describing LC states will be less than (2), since some of the transformations 
included in (1) for a given party will transform pure states to pure states and so are double-counted in (2). However for the purposes of this letter, we only require an upper bound and rate of growth with $n$ and $d$ of the number of parameters describing LC states and so (2) is adequate.

The above calculations show that for $n>2$, not all density matrices can be produced by local contamination of pure states (i.e. not all states are LC states). Specific examples are given below. We also note that in the limit of large $n$ we may say that the dimension of LC states is essentially the same as that of pure states, at least when compared to the dimension of the space of all states. Put another way, for large $n$, the space of LC states is exponentially smaller than the space of all mixed states.

However it is worth noting that for $n$ equal to two, the rate of growth of the number of parameters describing local contamination is $d^{4}$, as is the rate of growth of the number of parameters describing density matrices. It would be interesting to know whether every density of two $d$-level systems can arise as the result of local contamination of pure states. Note that, in order for it to be possible to reach an arbitrary target density matrix by local contamination of a pure state, it is necessary that the number of parameters describing pure states plus contamination be greater than or equal to that describing density matrices. However even if parameter counting allows it, this does not guarantee that it is indeed possible to reach an arbitrary target density matrix; a more refined analysis is required to determine this.

We now turn to the situation where the parties actively try to produce a target density matrix; in other words we allow measurements and classical communication. Our arguments will not rely on the counting of parameters which is much more subtle here. To see that there is an issue, consider the simple case of two $d$-level systems. We can easily show that using classical communication and local operations, Alice and Bob can produce an arbitrary density matrix. For let us write the target density matrix as a mixture of (typically entangled) pure states $\left|\psi_{\mu}\right\rangle$ :

$$
\rho=\sum_{\mu} p_{\mu}\left|\psi_{\mu}\right\rangle\left\langle\psi_{\mu}\right| .
$$

This state may be produced by the following protocol. Alice and Bob start with a maximally entangled state; we will use this pure state as the precursor for all target mixed states. We then simply note that any of the pure states $\left|\psi_{\mu}\right\rangle$ can be produced with probability one from the maximally entangled state by coordinated actions by

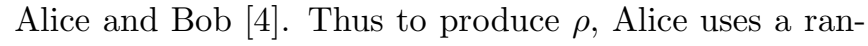
dom variable to produce an outcome $\mu$ with probability $p_{\mu}$; she communicates the value she receives to Bob; when they get the value $\mu$ they then perform the protocol to produce $\left|\psi_{\mu}\right\rangle$ with probability one from the maximally mixed state. Thus overall they have produced the required density matrix $\rho$. An optimised protocol, in the sense of using less entanglement, has been given recently by Vidal 5 .

Thus with classical communication Alice and Bob can produce any density matrix of two $d$-level systems (i.e. every two party state is LCCC). However as we now show, for more parties, in general, even with classical communication, not every density matrix arises as the local contamination of a pure state. For consider the following state of three qubits

$$
Z=p|\mathrm{~W}\rangle\langle\mathrm{W}|+(1-p)| \mathrm{GHZ}\rangle\langle\mathrm{GHZ}|
$$

where $|\mathrm{W}\rangle=\frac{1}{\sqrt{3}}(|001\rangle+|010\rangle+|100\rangle)[6]$, and $|\mathrm{GHZ}\rangle=$ $\frac{1}{\sqrt{2}}(|000\rangle+|111\rangle)$. Purifications of this state have the form

$$
|\Psi\rangle=\sqrt{p}|\mathrm{~W}\rangle\left|f_{1}\right\rangle+\sqrt{1-p}|\mathrm{GHZ}\rangle\left|f_{2}\right\rangle,
$$

where $\left|f_{1}\right\rangle$ and $\left|f_{2}\right\rangle$ are orthonormal states of the three ancillas (at this stage we have made no restrictions about these states; they might be entangled states of the three local ancilla Hilbert spaces). If it were possible to create the mixture by local degradation of a pure state then there must be a pure state $|\Phi\rangle$, such that adding local ancillas and evolving with local unitaries achieves the following transformation

$$
|\Phi\rangle|0\rangle \longrightarrow \sqrt{p}|\mathrm{~W}\rangle\left|f_{1}\right\rangle+\sqrt{1-p}|\mathrm{GHZ}\rangle\left|f_{2}\right\rangle .
$$

Since $\left|f_{1}\right\rangle$ and $\left|f_{2}\right\rangle$ are orthogonal, these two states may be distinguished with certainty using only local operations and classical communication [7]. Thus doing the measurement which distinguishes $\left|f_{1}\right\rangle$ from $\left|f_{2}\right\rangle$ collapses the state onto $|\mathrm{W}\rangle$ or $|\mathrm{GHZ}\rangle$ thus giving some non-zero probability of creating either $|\mathrm{W}\rangle$ or $|\mathrm{GHZ}\rangle$ by LOCC starting with $|\Phi\rangle$. However, it has been shown that the three-party states which may be converted with some probability into a $|\mathrm{GHZ}\rangle$ state by LOCC, and those which may be converted into a $|\mathrm{W}\rangle$ state form two disjoint classes 66]. Therefore we have a contradiction and so it is impossible to make the mixture $\rho$ in this way.

We note that our arguments apply equally well to any state of the form (4) where instead of $|W\rangle$ we have any state in the $|\mathrm{W}\rangle$ class and instead of $|\mathrm{GHZ}\rangle$ we have any state in the $|\mathrm{GHZ}\rangle$ class. In general, for larger numbers of particles, there may be a number of non-trivial inequivalent classes of entangled pure states, and we can produce non-LCCC states in a similar way. However the above argument will generalise only for mixtures of two different inequivalent pure states. This is because it has only been shown that two orthogonal states can be locally distinguished with certainty. Locally distinguishing more than two states may require the use of more copies [7].

The details of what happens in the case of more parties and higher spins remain to be worked out, although the general messages should be clear. In the case of no 
classical communication, typical density matrices cannot be produced by local contamination of pure states. In the case where we allow classical communication, it is certainly the case that any mixture of two pure states falling into disjoint classes (as with $|\mathrm{W}\rangle$ and $|\mathrm{GHZ}\rangle$ in the case of three qubits) will give rise to a "complex" density matrix (ie. one which cannot be formed by local contamination of a pure state). This is a particular method for constructing non-LCCC density matrices; we suspect that there are many other interesting classes of non-LCCC states.

The main goal of this letter was to raise the question of how density matrices can arise. Once the question has been raised, many other interesting issues suggest themselves. A first obvious one is to find a method for characterising whether a given mixed state is $\mathrm{LC}$ or LCCC. More generally we would like to understand how the space of all states decomposes into classes of states which are accessible from each other by local degrading (with or without classical communication). We would also like to characterise these classes by their entanglement properties and assess their physical implications. Finally, the fact that noise only takes pure states into a limited range of mixed states, rather than to the whole space of mixed states (of exponentially larger dimensionality), offers a quite new perspective on quantum error correction [8].

\section{Acknowledgments}

We gratefully acknowledge funding from the European Union under the project EQUIP (contract IST-199911063).

[1] The number of recent papers dedicated to the study of density matrices is very extensive. A necessarily incomplete list is: R. F. Werner, Phys. Rev. A 40, 4277 (1989), L.P. Hughston, R. Jozsa and W.K. Wootters, Phys. Lett. A 183, 14 (1993), S. Popescu, Phys. Rev. Lett. 74, 2619 (1995), A. Peres, Phys. Rev. Lett. 77, 1413 (1996), M. Horodecki, P. Horodecki and R. Horodecki, Phys. Lett. A 223, 1 (1996), C.H. Bennett, G. Brassard, S. Popescu, B. Schumacher, J.A. Smolin and W.K. Wootters, Phys. Rev. Lett. 76, 722 (1996), N. Linden, S. Massar and S. Popescu, Phys. Rev. Lett. 81, 3279 (1998), W. K. Wootters, Phys. Rev. Lett. 80, 2245 (1998), M. Horodecki, P. Horodecki and R. Horodecki. Phys. Rev. Lett. 80, 5239 (1998), V. Vedral and M. B. Plenio, Phys. Rev. A 57, 1619 (1998), G. Vidal and R. Tarrach, Phys. Rev. A 59, 141 (1999), S. Braunstein, C.M. Caves, R. Jozsa, N. Linden, R. Schack and S. Popescu, Phys. Rev. Lett. 83, 1054 (1999), N. Linden, S. Popescu and A. Sudbery, Phys. Rev. Lett. 83, 243 (1999), C. H. Bennett, D. P. DiVincenzo, T. Mor, P. W. Shor, J. A. Smolin and B. M. Terhal, Phys.
Rev. Lett. 82, 5385 (1999), P. Horodecki, M. Horodecki and R. Horodecki, Phys. Rev. Lett. 82, 1056 (1999). Many references to recent literature may also be found in M. Lewenstein, D. Bruss, J.I. Cirac, B. Kraus, M. Kus, J. Samsonowicz, A. Sanpera, R. Tarrach, J. Mod. Opt. 47, 2841 (2000), M.A. Nielsen and I.L. Chuang, Quantum Computation and Quantum Information, (CUP, Cambridge, UK, 2000).

[2] In the case of LCCC, if we allow pure state precursors of higher dimensionality than the target mixed state, then any mixed state can be trivially obtained: for example by arranging for maximally entangled states between all pairs of parties, producing the required mixed state locally at one particular location, and then using teleportation.

[3] B. Schumacher, Phys. Rev. A, 54, 2614 (1996).

[4] M.A. Nielsen, Phys. Rev. Lett. 83, 436 (1999).

[5] G. Vidal, Phys. Rev. A 62062315 (2000).

[6] W. Dür, G. Vidal and J. I. Cirac, Phys. Rev. A 62, 062314 (2000).

[7] J. Walgate, A. J. Short, L. Hardy and V. Vedral, quantph/0007098 (2000).

[8] P.W. Shor, Phys. Rev. A 52 R2493 (1995), A.M. Steane, Phys. Rev. Lett. 77793 (1996). 